\title{
Arbor
}

\section{Las transiciones de la familia española}

\section{Salustiano del Campo.}

Arbor CLXXVIII, 702 (Junio 2004), 451-464 pp.

Transición es el paso de un estado o situación a otro, y el término se aplica a todos los fenómenos y seres vivos que experimentan cambios. Según el diccionario es «cambio o mudanza de un modo de ser o estar a otro distinto». Obviamente, el término transición es aplicable a la familia que, aunque en un tiempo determinado se concebía en nuestro país como exenta de cambios, hoy se reconoce como sujeta a múltiples transformaciones. De ella me voy a ocupar a continuación haciendo hincapié de la reversibilidad o irreversibilidad de sus transiciones. ¿Son o no definitivas? ¿Todas o solamente algunas? ¿Ninguna, quizás? Mi objetivo es, sobre todo, poner de manifiesto cómo redefinen las transiciones a una institución tan omnipresente, en el tiempo y en el espacio, como la familia.

No se debe olvidar, sin embargo, que en la evolución social, como oportunamente advirtió Augusto Comte, todo lo que no se sustituye sobrevive $y$, por tanto, la pregunta clave es si habrá en el futuro uno o más tipos de familia. Esto es, si marchamos hacia un único modelo o nos alejamos de él. La realidad parece indicar que la antigua hegemonía del modelo de familia extensa, primero, y de familia nuclear después, no tiene un gran futuro, cuanto menos a corto plazo.

\section{La teoría clásica de la transición demográfica}

La teoría de la transición demográfica que podemos llamar clásica la formuló Frank Notestein en $1945^{1}$ y nos sirve para iniciar la consideración de las transiciones de la familia que es, desde el punto de vista analítico, una institución mediadora 0 , si se quiere, la variable institucional 


\section{Salustiano del Campo}

más relevante en el engarce del individuo con la sociedad, incluso en el tránsito de la sociedad tradicional a la sociedad moderna.

Como es sabido, esta teoría se fundamenta a escala mundial en la evolución de la mortalidad y de la natalidad desde una situación estable en un nivel alto -a lo largo de los muchos siglos de sociedad tradicionala otra de nivel bajo y también estable, tras atravesar una etapa desequilibrada de crecimiento explosivo en la que primero desciende la mortalidad por el desarrollo económico, por la sanidad ambiental y por la medicina moderna y más tarde lo hace la natalidad cuando se dispone de anticonceptivos seguros y fiables.

David Riesman en su famosa obra La muchedumbre solitaria ${ }^{2}$ dedujo, a partir de esta teoría, la evolución del carácter individual que sintetizó en tres tipos: dirigido por la tradición, autónomo y heterodirigido. A partir de estos conceptos analizó y explicó otros cambios sociales que acompañan a este tránsito, pero no es esta ocasión para comentar ni siquiera someramente el contenido de su obra.

La última fase de la transición de las sociedades industriales avanzadas, que es en la que nos encontramos, por diversas razones se denomina también postmoderna, postindustrial, sociedad del conocimiento, sociedad de servicios, sociedad de la información y de varias otras maneras.

\section{La segunda transición demográfica}

Si la primera o clásica transición demográfica fue producto, y acarreó, grandes cambios estructurales en la sociedad humana, como la industrialización, la urbanización, la secularización la alfabetización y otros, la que se llama «segunda transición demográfica» ha sido y es eminentemente cultural y empezó a percibirse cuando en los años 70 se difundió la conciencia de la que Inglehart ha calificado de "revolución silenciosa ${ }^{3}$, que es el antecedente de la formulación teórica desarrollada en los años 80 y 90 por demógrafos holandeses (Lesthaege y otros) ${ }^{4}$. Dentro ya de la etapa de baja natalidad, unos cambios complejos y todavía no bien conocidos conducen a un desplome de la natalidad por debajo del nivel de reemplazamiento de las generaciones, hasta el punto de que éste es hoy el caso de todas las naciones europeas, mientras que en los años 60 todas tenían tasas de fecundidad superiores a 2,1 hijos por mujer.

Este parece ser el punto final de la desaparición de la fecundidad regida por normas sociales y el paso pleno al predominio del individualis- 
mo en la actividad reproductora, reforzado además por la anticoncepción y por las modernas tecnologías de la reproducción, desde la fecundación in vitro hasta la clonación cuando llegue. En este periodo la anticoncepción está sometida al dominio de la mujer porque los métodos anticonceptivos que se aplican están centrados en ella.

Como es lógico suponer, todo esto ha influido de modo importante en la familia, primordialmente a través de los cambios en la fecundidad y del nuevo status de la mujer, pero su efecto total es tan grande que requiere una consideración separada de la serie de transformaciones específicas que afectan de lleno a la familia. Por esto, en las páginas que siguen consideraré diez muy significativas, si bien este número no es limitativo.

\section{Las transiciones familiares}

\section{Pérdida de centralidad de la familia}

En el tránsito de la sociedad tradicional a la sociedad moderna la familia ha pasado de ser la institución primordial a ser una más entre otras, como se ha glosado muchas veces. La familia, que en otros tiempos ejercía una enorme cantidad de funciones, se ha transformado actualmente en una institución especializada con unas pocas funciones propias, que a menudo se identifican con ser sede de afectos y agente socializador de los individuos.

Naturalmente, este tránsito no ha seguido una evolución normal, sino que es una consecuencia de los acontecimientos que pusieron fin al antiguo régimen. Hace algún tiempo el sociólogo James Coleman dedicó un interesante artículo a la reconstrucción racional de la sociedad ${ }^{5}$, una vez que el orden social preexistente fue destruido por la revolución francesa y por los múltiples acontecimientos y cambios que la acompañaron y siguieron. De este modo, la familia como protagonista universal de las funciones esenciales para la sociedad fue sustituida por la gran corporación y por el Estado. Aquella le aportaba continuidad, puesto que no era tan perecedera como la vida humana y el segundo le ofrecía un método de acción absolutamente racional como es la burocracia. Se transita así desde la organización social primordial basada en lazos de sangre a una organización social construida deliberadamente. Antes, la estructura social descansaba en la familia, mientras que ahora sus agentes principales pasan a ser personas jurídicas, que duran más que los individuos, como lo hacía y sigue haciendo la familia. 
Esta descripción de Coleman coincide con el propósito de reconstruir el orden social que animó a Augusto Comte a fundar la Sociología y en el proceso subsiguiente la comunidad se sustituyó por la sociedad, como observó agudamente Ferdinand Tonnies y la gran corporación adoptó la forma de gestión burocrática. El control sobre los individuos que ejercían la familia y la Iglesia pasaron a las manos de las corporaciones y del Estado, pero no sin un coste elevado.

Este proceso implicó una descapitalización de la sociedad que aún no ha sido compensada adecuadamente. Ahora bien, ¿este empobrecimiento de la familia, o esta pérdida de funciones, supone quizás su desaparición? ¿Tal vez su eclipse, al menos parcial? ¿Qué, si no? Muchos autores han coincidido en calificar como de pérdida de funciones de la familia la secuencia temporal que hemos descrito (Ogburn, Nimkoff y Burgess). Esta visión que se ha convertido en la dominante no es, sin embargo, capaz de explicar adecuadamente lo que de verdad ha pasado en la familia y por eso en un trabajo mío he traído a colación el concepto de deconstrucción, con el cual se explican mejor, a mi parecer, la diversificación actual de los tipos de familia, la evolución de los modelos matrimoniales, la desinstitucionalización de la familia nuclear y una serie de disociaciones de algunas de las cuales me ocuparé en las páginas que siguen.

Pero hay una observación que es procedente aquí y que contradice el modelo forjado sobre la noción de pérdida de funciones. Se trata del hecho inesperado de que, incluso en una situación en la que la familia española ha estado casi totalmente desprotegida por los poderes públicos, ha atraído hacia sí el desempeño de nuevas funciones relacionadas con la pésima situación de la gente joven. Ha sido la familia la que la ha atendido en los casos de drogadicción, paro, fracasos matrimoniales y otros, una vez concluida la rebelión contra la generación anterior protagonizada por los jóvenes sensentayochistas.

\section{Desinstitucionalización}

El matrimonio, desde la vieja Roma y las Decretales de Graciano, es una construcción social basada en un capital social acumulado. Por desinstitucionalización debe entenderse aquí la reducción del estado de la institución, hasta situaciones de facto que desempeñan cometidos semejantes, si no idénticos, como el paso del matrimonio a la unión consensual. No es de ninguna manera el descenso de la natalidad, como algunos frívolamente suponen. 


\section{Las transiciones de la familia española}

Casos recientes son la desaparición en los códigos y demás textos legales de los requisitos de la filiación legítima que en otros tiempos fueron muy prolijos y detallados. De reglas vigentes durante siglos se eliminan formalidades $\mathrm{y}$ se pierden elementos importantes e incluso se aprecia una tendencia a centrar el análisis demográfico y sociológico en los hogares y no en las familias, al estilo como se hacía en tiempos medievales y con la excepción de los hogares unipersonales que, por definición, no son familia. De este modo, poco a poco se invisibiliza a la familia y los poderes públicos se aprovechan de ello para negarle importancia económica, política y cultural y devolverle el poder sobre los hijos que cedió a las escuelas cuando hubo de hacerse obligatoria la educación primaria, porque los padres se resistían a renunciar a la ayuda material de sus hijos para hacer frente a los trabajos del año agrícola.

Con el paso de la sociedad agraria y tradicional a la sociedad industrial y moderna pierden relevancia los grandes sistemas familiares del pasado, como ha demostrado Emmanuel Todd ${ }^{6}$ y también determinadas instituciones de derechos forales que producen ahora efectos inversos a los que estaban destinadas a cumplir. Los segundones de la familia catalana tradicional se han visto favorecidos inesperadamente por la revalorización de las tierras marginales y poco útiles para la labranza y la productividad agrícola y, sobre todo, por los terrenos costeros, que se les asignaban con objeto de que el primogénito (el hereu) conservara unidas las mejores tierras cultivables.

En definitiva, lo que en este epígrafe se contempla es el paso de la familia de institución pública a institución privada, y paralelamente la sustitución del modelo único de la familia tradicional, que estuvo vigente durante tantos siglos, por la actual variedad de formas de familia.

\section{De institución cerrada a institución abierta}

Según ha mostrado el sociólogo Louis Roussel ${ }^{7}$, cada modelo de matrimonio se completa hoy con su propio modelo de divorcio. Los cuatro tipos de matrimonio que él define están relacionados con la presencia o no en ellos del divorcio. El matrimonio institucional es cerrado y no admite el divorcio, pero sí lo hacen el matrimonio alianza, el matrimonio fusión y el matrimonio asociación.

El modelo de matrimonio alianza se completa con un modelo de divorcio en el que hay culpable y, por tanto, sanción. No pasa eso con el matrimonio fusión en el cual no hay culpable y, por supuesto, tampoco con 
el matrimonio asociación, que roza los límites del simple pacto para el intercambio temporal de servicios sexuales y de compañía.

Progresivamente han ido imponiéndose la voluntad de las partes y las cláusulas acordadas entre ellas, de modo que la intervención de la sociedad queda reducida a una función de arbitraje, que poco tiene que ver con la familia romana. El afán de privatización de su relación por los futuros cónyuges ha hecho que la familia pierda buena parte de su status público y, al hacerlo por la presión de determinados grupos como los feministas, se provoca la paradoja de que pretenden privilegiar el contrato privado que se crea por el consentimiento de las partes sobre cualquier otro contrato bilateral. Con frecuencia se reclama que el Estado pague los alimentos a las madres divorciadas con hijos, pero se sigue impugnando la legitimidad institucional de la familia. No es el momento mejor pero no hay que olvidar aquí que el divorcio, frente a lo que se cree, no rompe la familia sino nada más el vínculo que une a los cónyuges o a la pareja, mientras que los demás subsisten.

\section{La pluralidad de las formas familiares}

Aunque en cada etapa de la evolución social han coexistido formas mayoritarias y minoritarias de familia, la preeminencia de un tipo ha sido una constante y con carácter general se puede afirmar que tanto en los pueblos primitivos cono en las sociedades industriales avanzadas el tipo de familia predominante ha sido el nuclear o conyugal, compuesto por padre, madre e hijos. Hoy todavía no hay ningún país europeo en el que este tipo de familia sea inferior al $50 \%$ del total, pero dentro de las formas minoritarias hay algunas que tienen un volumen importante.

Para estudiar la pluralidad de formas de familia es preciso antes referirse a los tipos de matrimonio, como ha hecho el demógrafo francés Louis Rousel. No se trata simplemente de que como establecen nuestra Constitución, y se practica en la mayoría de las sociedades de nuestro entorno, la forma de contraerlo pueda ser religiosa o civil, sino de que hay claras diferencias entre los cuatro tipos de matrimonio que él analiza a los que me he referido anteriormente.

Por otro lado, aunque en el momento presente hay algunos tipos de convivencia familiar a parafamiliar que en otros tiempos tuvieron un cierto auge, como las comunas, los matrimonios de grupo, los matrimonios LAT, etc., los tipos de familia más importantes del momento actual son: la conyugal o nuclear, a la que me he referido antes, la monoparen- 
tal y la recompuesta ${ }^{8}$. La monoparental consta de un padre o una madre que viven con sus hijos y, para ser exactos, aproximadamente el $90 \%$ de ellas están encabezadas por mujeres. A su vez, la familia recompuesta está formada con restos de otras familias, es decir, consta de una pareja que ya ha participado en una unión anterior y cuyos hijos tenidos en ella se aportan a la nueva, más los hijos propios de ambos cónyuges. Debido sobre todo a la incidencia del divorcio en unos casos, y a los embarazos de adolescentes, las familias monoparentales abundan en las sociedades de nuestro tipo, aunque en España no alcanzaban en 1991 ni siquiera el $11 \%$. En cuanto a las recompuestas no llegan entre nosotros al $4 \%$ si bien no pocos autores la consideran el tipo de familia de futuro. No sobra advertir en este punto que la confusión entre matrimonio y familia conduce a veces a aceptar como formas de familia lo que son fundamentalmente formas alternativas de ella, como los hogares unipersonales y la cohabitación o el concubinato, que se está convirtiendo en una forma muy general de convivencia.

Por definición, una persona que vive sola no es una familia, aunque conserve las relaciones familiares con sus parientes y, a su vez, el celibato o la unión consensual no formalizada tampoco es propiamente hablando una familia. En este último caso, sin embargo, hay que hacer la salvedad de que cuando este último tipo de familia dura un cierto tiempo -una década o más- los efectos de su ruptura se asemejan mucho a los de un matrimonio de idéntica duración y tampoco hay que olvidar que formalmente los Common Law Marriages de los países anglosajones no son otra cosa que uniones consensuales a las que se concede socialmente la presunción de matrimonio por su duración y por su respetabilidad. La variedad de formas de familia, tanto de los mencionadas como más importantes como de las demás, se califica a menudo un tanto despectivamente como menú de familias. Parece como si desde la situación de cualquier sujeto se pudiera elegir la que se desee, sin tener en cuenta que algunas requieren atravesar una condición anterior indispensable. Así, para ser divorciado se requiere haber estado casado y para ser madre divorciada de familia monoparental se requiere haber roto por lo menos un matrimonio anterior.

\section{De la desigualdad a la igualdad}

La familia es una institución internamente desigual al estar fundada sobre los criterios adscritos del sexo y de la edad. En cuanto a esta última, es obvio que la división simple entre la infancia, la juventud, la madurez y 
la vejez asigna diferentes roles a cada una de las edades y una sujeción real o dependencia, desde muchos puntos de vista, de unos individuos a otros.

Por otro lado, la situación de preeminencia del varón en la familia tiene antecedentes muy remotos y, en la fase de familia, burguesa, está basada en la atribución de la representación de la familia y del papel ganador del sustento familiar al marido, así como en la relegación al interior del hogar de la mujer. Frente a eso, las modernas leyes de matrimonio establecen la igualdad entre los cónyuges, que comparten la patria potestad y tienen derechos y deberes recíprocos. En términos generales podemos decir que la relación entre los cónyuges ha tendido desde el siglo XIX a la igualdad, al equilibrio y a la solidaridad. El caso de los hijos no es el mismo, pero evidentemente se independizan bastante pronto, la mayoría de edad se ha adelantado y las leyes actuales limitan el poder de los padres. Su autoridad sobre los hijos se reduce, si no es que desaparece, y la tendencia parece intensificarse por las progresivas rebajas de la mayoría de edad legal, de la edad de votar, de la edad de responsabilidad legal, y de otras.

El fundamento de la igualdad de los cónyuges dentro del matrimonio procede de lo que acontece fuera de él y responde a la participación de la mujer en la actividad productiva, que repercute en su condición en el interior del matrimonio. El caso de la familia donde tanto el marido como la mujer tienen sus propias carreras es paradigmático de esta situación.

\section{De la autonomía de la familia a la autonomía personal}

En la familia tradicional, uno de cuyos principales objetivos era la transmisión del patronímico y del patrimonio, la dinámica interior de las relaciones entre los miembros apenas tenía reflejo en el exterior. Se trataba de asuntos íntimos que raramente veían la luz, hasta el punto de que la fachada de la familia era las más de las veces impenetrable. El honor del nombre se imponía y contrastaba con la facilidad que hoy se tiene para cambiar de nombre o dejar de usar el que se lleva.

El tránsito de la familia tradicional a la familia moderna se apoya inicialmente en la libre elección del cónyuge y en que poco a poco van pesando cada vez más los elementos de atracción personal que se sobreponen a los del bien de la estirpe o de la familia.

Su culminación llega cuando el elemento principal para la formación de la familia pasa a ser el ideal de felicidad de los cónyuges, y hasta tal punto es esto así que el historiador francés P. Ariès ha señalado que la 


\section{Las transiciones de la familia española}

etapa de la familia moderna en la cual el centro era el niño ha cedido ya el paso al reinado de la pareja que destrona al niño, como se refleja en la literatura.

\section{La desvinculación de la sexualidad del matrimonio y la procreación}

Hasta hace unas cuantas décadas la gran mayoría de las relaciones sexuales que se mantenían en las sociedades se daban dentro del matrimonio, siendo las relaciones prematrimoniales y las extramatrimoniales categorías casi residuales, estadísticamente hablando.

Ahora la sexualidad ha cambiado y se ha constituido como un valor o un bien en sí mismo ${ }^{9}$. A esto han contribuido la participación de la mujer en el espacio del trabajo fuera del hogar, que antes era dominio casi exclusivo del hombre. De esta manera, la asequibilidad del otro sexo se ha convertido en universal, a despecho de la condición civil de la persona implicada, dado que los matrimonios pueden disolverse voluntariamente. $\mathrm{Al}$ mismo objeto han contribuido la seguridad y fiabilidad de los anticonceptivos disponibles desde los años 60 , que liberan casi totalmente a la mujer del temor del embarazo y, por último, el hecho a menudo olvidado de que los actuales anticonceptivos están controlados por la mujer, habiendo perdido el hombre su anterior papel central en la anticoncepción. De hecho, los métodos clásicos del varón, el retiro y el uso del preservativo, son actualmente bastante minoritarios en las sociedades industriales avanzadas.

El conjunto ha contribuido, como se ha indicado, al cambio de status de la mujer y ha aportado nuevos matices a la perenne batalla de los sexos, cuyas modalidades y resultados difieren bastante en nuestro continente entre las sociedades escandinavas y las mediterráneas.

También hay que tener en cuenta en este punto las llamadas tecnologías de la reproducción, que han hecho posible por primera vez en la historia que nada menos que cinco personas intervengan en el nacimiento de un nuevo ser y ponen al alcance de la mano la posibilidad de clonar seres humanos con propósitos reproductivos.

\section{De los servicios familiares a los servicios sociales}

Entre las múltiples funciones desempeñadas por la familia a lo largo de la historia ha sido fundamental la de la solidaridad familiar, tanto en- 
tre los cónyuges como entre los padres y los hijos y entre los propios hermanos. En cierto modo, esta ayuda formaba parte de la centralidad de la familia extensa en la vida de los individuos. Hoy, sin embargo, las sociedades industriales avanzadas son sociedades de servicios, en las que la satisfacción de las necesidades de los individuos se cumplen por lo general a través de instituciones especializadas. Algunos autores distinguen entre las corrientes genéricas de ayudas intrafamiliares, separando las ayudas financieras y las de la realización de las tareas domésticas. La protección social en las sociedades de servicios puede obtenerse de distintas maneras: a través de la familia, como ha sucedido tradicionalmente; a través del Estado de Bienestar, que es propio de nuestra época; a través del mercado, donde se puede comprar ${ }^{10}$.

Recientemente, una comparación entre las modalidades de protección social en las sociedades nórdicas y en las mediterráneas ha puesto de manifiesto que no siempre la superioridad está de parte de las sociedades en las cuales la protección proviene del Estado y del mercado. En realidad, los países mediterráneos se caracterizan por depender más de la protección familiar y los países nórdicos por el predominio de la protección propia del Estado de Bienestar, pero lo característico de todos es el Welfare Mix.

\section{La revolución de las edades}

A lo largo de la historia de la humanidad lo que hacían los jóvenes, los maduros y los viejos estaba bastante definido en todas las sociedades. Una fase de aprendizaje enseñaba lo que había que hacer en la edad adulta y, finalmente, los viejos se retiraban y servían a su grupo mediante el consejo y el prestigio acumulado. Una nueva versión de las etapas de la vida, de la que han hablado autores tan importantes como Goethe o Shakespeare, es en la moderna Sociología funcionalista la identificación de ciertos ritos de paso que introducen a la edad adulta. Así, Parsons señala que la finalización de los estudios, el primer empleo y el matrimonio son lo que hacen adulto a un hombre y a una mujer en nuestras sociedades. A pesar del poco tiempo transcurrido desde esta última formulación, las cosas han cambiado mucho debido a causas tan diversas como el aumento de la esperanza media de vida, o las dificultades de encontrar empleo o la mayor riqueza de los viejos comparados con los jóvenes.

Por un lado, actualmente conviven más generaciones que nunca y Reuben Hill pudo hablar ya de la familia de cinco generaciones, lo que 
pasa es que con esto ciertas instituciones que tenían una gran importancia en la familia la han disminuido o perdido, como pasa con la herencia. También la juventud se ve afectada por las dificultades de empleo o su precariedad y alarga el tiempo de la dependencia de su familia de origen, hasta el punto de que se puede hablar de una prolongación forzada de la adolescencia. En el caso de España, que tiene la edad de emancipación más tardía de todo el continente europeo, esto es bastante claro.

La madurez, por su parte, ha sido la fase de la vida en la que se ha ejercido el poder social y familiar, sobre todo, cuando los trabajos eran fijos y abundaba el pleno empleo. Hoy, parte de ella por la fuerza de las circunstancias, se ha convertido más bien en la edad de los reciclajes profesionales y de la prejubilación.

Y nos queda la vejez, que algunos quieren paradójicamente considerar como la edad de la esperanza. Sin duda sigue siendo muy difícil responder la siguiente pregunta: ¿Es la vejez el filón cuyo producto salvará nuestra sociedad? Por el momento no es fácil contestarla, si bien la vejez cada día aparece menos como un bloque compacto y ganan en importancia sus subdivisiones interiores. Más bien la vejez parece ser la asignatura pendiente de la familia y por extensión de la sociedad, aunque hay otros problemas derivados también de la edad que no pueden ignorarse, como son la desaparición de la fraternidad y la revalorización de los abuelos.

\section{De la familia como sede de relaciones generacionales a serlo de relaciones intergeneracionales}

Antes se ha señalado cómo hace años ya Reuben Hill habló de las familias de cinco generaciones y sobre esta plantilla se modeló un nuevo ciclo de vida, pero hay otras características de la familia actual que también deben tenerse en cuenta en este punto. Las 2 ó 3 generaciones que antes estaban en contacto en el interior de la familia se han ampliado a 5 , pero también la familia ha cambiado su horizontalidad de otros tiempos por su verticalidad actual. Hoy, con 1 ó 2 miembros por generación, se ha hecho filiforme y, dada la longevidad de sus miembros, el envejecimiento ha ganado en importancia. En este punto y para no abandonarlo sin ninguna otra consideración, cabe observar que si en la vida social las comunidades no se forman verticalmente, esto puede no ser aplicable en el territorio de la familia y, por otro lado, es lícito preguntarse si el paso de las relaciones intrageneracionales a intergeneracionales enriquece $o$ no a la familia. No parece que esto último sea cierto si tenemos en cuen- 
ta el empobrecimiento que representa la desaparición a todos los efectos prácticos de la fraternidad.

\section{Observaciones finales}

Y termino con algunas consideraciones y bastantes interrogantes. El primero se refiere a la continuidad y discontinuidad en la evolución familiar que históricamente nos aparece incierta: ¿hacia donde marcha la evolución familiar? Cuando se alcanza un nuevo estadio estas preguntas tienen diferentes respuestas, aunque los intentos de contestarlos por parte de los historiadores de la familia no escasean y son importantes.

En segundo lugar, la familia no es hoy como lo fue en la Roma clásica, institución de derecho público. En Roma era valiosa porque en ella se tenían los hijos y hoy se tienen pocos hijos y, además, no es imprescindible para engendrarlos. Algunos autores apuntan incluso a la posibilidad de que en nuestras sociedades se encomiende el papel de reproductoras a un grupo de mujeres especializadas. A todo esto, no se puede olvidar que contemporáneamente existe una impugnación familiar universal contra la familia, aunque el antifamilismo ya no sea ni tan pronunciado ni tan burdo como lo fue en los albores del feminismo. Sin embargo, está hecho de parcialidades, mixtificaciones, tergiversaciones, malas interpretaciones y otras prácticas que hay que desvelar mediante un trabajo serio y objetivo.

Hubo un tiempo en el que se afirmaba que la diferencia entre una revolución y un simple golpe de Estado consistía en que la primera aspiraba a cambiar la familia. En la actualidad, la pérdida de la centralidad, de la que antes hemos hablado, la ha desmontado también de este pedestal. Por otra parte, sigue siendo un enigma el papel que la religión juega actualmente en la familia. El profesor Javier Elzo ha recordado que la evolución de la familia se produce casi totalmente el margen de la religión, aunque dentro de lo que él llama un humus de catolicidad ${ }^{11}$. A mi juicio, este es un gran tema que hasta aquí no ha sido tratado de un modo consecuente y adecuado. Los sentimientos que se identifican con ella, como la lealtad, la fidelidad, el amor fraterno y otros, a menudo son de cuño religioso y otros sentimientos son igualmente ensalzados por la religión, como el instinto maternal, pero no hemos conseguido ir más allá de esta conclusión tan general y hay un último punto al que también es preciso referirse. Se trata de averiguar cómo y en qué medida afectan las políticas familiares a la evolución de la familia. 
Como se sabe, las políticas familiares pueden ser explícitas o implícitas, de modo que las hay incluso allí donde no se reconoce su presencia. Carecer de política familiar es simplemente una forma de tenerla, pero nuestra Constitución en su artículo 39 ordena que exista y las Naciones Unidas atribuyen su autoría exclusivamente a los Estados y no a las instancias supraestatales. El plan de acción mundial en materia de población fue adoptado por todas las naciones desde la Conferencia Mundial de Bucarest de 1984, pero no hay una política europea de la familia al margen o por encima de los Estados.

Curiosamente, el efecto de la política familiar cuando es manifiesta es desigual en los diferentes campos que abarca. Es visible y efectiva en el caso de la inmigración, donde funcionan permisos de trabajo y residencia, visiones de la multiculturalidad e integración y otras. También en el empleo, que reviste distintas modalidades: fijo, parcial, a tiempo completo, precario y otros. Es plenamente eficaz en el caso de la mortalidad, porque se formulan medidas para combatir enfermedades de nuestro tiempo, como el SIDA o el SARS, y de dudosa eficacia cuando se trata de la natalidad. Nunca se ha comprobado que las políticas natalistas eleven significativamente la natalidad y lo común es que su efecto, aunque positivo, sea mínimo.

\section{Conclusión}

Y quiero terminar volviendo al principio. He procurado reflejar los cambios de una realidad dinámica, pero me he cuidado de atribuirles una condición permanente. Tampoco me parecen efímeros y, en todo caso, lo que son es importantes. A mi juicio, revelan una vez más el esfuerzo de la familia por adaptarse a las condiciones actuales como lo ha hecho a lo largo de la historia. Así es como ha ganado y garantizado su supervivencia y seguimiento y puede afirmarse que pasará una vez más; por lo menos, pronosticarlo es plausible y probable que se realice.

\section{Notas}

1 Frank W. Notestein in «Population - The Long View», in T. W. Schultz, Food for the World (Chicago: The University of Chicago Press, 1945).

2 David Riesman.: The Lonely Crowd. New Haven, 1950.

3 RONALD INGLeHART,: El cambio cultural en las sociedades industriales avanzadas, Madrid, Centro de Investigaciones Sociológicas, 1991.

4 D.J. VAN DE KAA: «Europe's Second Demographic Transition» Populaton Bulletin, vol. 42, num.1, marzo de 1987. Un año antes, R. Lesthaeghe y D. J. van de Kaa habían publicado un artículo en holandés titulado «¿Dos transiciones demográficas?» 


\section{Salustiano del Campo}

464

${ }^{5}$ | James S. Coleman: «The rational reconstruction of society», American Sociological Review, vol. 58, febrero 1993, pp. 1-15.

${ }^{6}$ Emmanuel Todd: La invención de Europa, Tusquets, Barcelona, 1995.

7 Louis Roussel: «Marriages et divorces. Contribution à un analyse systematique des modèles matrimoniaux", Population, 1980, noviembre-diciembre:1025-1040.

8 Véase Salustiano del Campo: Familias: Sociología y Política. Ed. Complutense, Madrid, 1995, pp. 43-48.

9 Ver mi libro, op. cit., pp.32-37.

10 Ver MARÍA TERESA LÓPEZ LÓPEZ: La protección social a la familia en España y en los demás Estados miembros de la Unión Europea, Fundación BBV, Madrid, 1996.

11 JAVIER Elzo: «Tipología de las familias españolas», ponencia en la 3. ${ }^{a}$ Sesión de la Escuela de Sociología de la Familia de la UIMP (en este mismo volumen). 